\title{
Epidemiological, clinical, and microbiological characteristics of carbapenemase-producing Enterobacteriaceae bloodstream infection in the Republic of Korea
}

\author{
Jung-wan Park ${ }^{1,2+}$, Hyungmin Lee ${ }^{2 \dagger}$, Se Yoon Park ${ }^{3 *}$ and Tae Hyong Kim ${ }^{3}$
}

\begin{abstract}
Background: Carbapenemase-producing Enterobacteriaceae (CPE) is an important pathogen in nosocomial infections; nevertheless, only a few studies regarding CPE infection and its epidemiological factors have been conducted in the Republic of Korea (ROK). We aimed to analyze the clinical, microbiological, and epidemiological characteristics of CPE bloodstream infections (BSIs) in the ROK.

Methods: This retrospective cohort study included data collected from the National Surveillance System from January 2015 to December 2016 based on the epidemiologic survey performed by an epidemiologist from the Korea Centers for Disease Control and Prevention. We selected patients with CPE BSI from the Korea National Institute of Health based on carbapenemase genotyping.

Results: In this study, 131 CPE BSIs were identified, and the proportion of CPE BSI among total CPE isolates was 7\%. Klebsiella pneumoniae accounted for $69 \%$ of all CPE BSIs, and $66 \%$ of these produced K. pneumoniae carbapenemase. Among nine provinces in ROK, one province had NDM as the most common carbapenemase. CPE was susceptible to amikacin, tigecycline, and gentamicin $(76,41$, and 39\%, respectively). Of 29 patients tested for colistin sensitivity, one patient showed colistin resistance. The most common CPE BSI sources were pneumonia, primary bacteremia, and biliary tract infection. Multivariable analysis showed that adequate antibiotic use at CPE detection was significantly associated with decreased 30-day mortality.
\end{abstract}

Conclusions: CPE BSIs are prevalent in the ROK. Moreover, most CPE BSIs originated from hospital-acquired infection, demonstrating the need to improve hospital infection control strategies.

Keywords: Infection, Carbapenemase-producing Enterobacteriaceae, Republic of Korea, Klebsiella, Nosocomial infection

\footnotetext{
* Correspondence: sypark@schmc.ac.kr

${ }^{\dagger}$ Jung-wan Park and Hyungmin Lee contributed equally to this work

${ }^{3}$ Division of Infectious Diseases, Department of Internal Medicine,

Soonchunhyang University Seoul Hospital, Soonchunhyang University

College of Medicine, 59, Daesagwan-ro, Yongsan-gu, Seoul 04401, Republic

of Korea

Full list of author information is available at the end of the article
}

(c) The Author(s). 2019 Open Access This article is distributed under the terms of the Creative Commons Attribution 4.0 International License (http://creativecommons.org/licenses/by/4.0/) which permits unrestricted use, distribution, and reproduction in any medium, provided you give appropriate credit to the original author(s) and the source, provide a link to the Creative Commons license, and indicate if changes were made. The Creative Commons Public Domain Dedication waiver (http://creativecommons.org/publicdomain/zero/1.0/) applies to the data made available in this article, unless otherwise stated. 


\section{Background}

Enterobacteriaceae is one of the major contributors to nosocomial infections. Klebsiella pneumoniae and Enterobacter species infections account for $15-51 \%$ of all nosocomial infections in intensive care units $[1,2]$. Furthermore, there has been a gradual increase in the risk for multidrug resistance among Enterobacteriaceae, including carbapenemase-producing Enterobacteriaceae (CPE) [3, 4].

Since the first case of CPE was reported in 2010 in the Republic of Korea (ROK) (5), the number of patients with $\mathrm{CPE}$ infection or those carrying CPE has increased exponentially. Several medical institutions have experienced CPE outbreaks due to transmission within healthcare settings [6]. With the rise in the number of CPE infection cases, the associated disease burden is also increasing [7]. The 30-day mortality of CPE bloodstream infection (BSI) is approximately $50 \%$, despite the application of empirical treatment [8]. The mortality associated with CPE infection is four times higher than that associated with non-carbapenemase-producing carbapenem-resistant Enterobacteriaceae (non-CP-CRE) [9].

Due to the high rate of proliferation and virulence associated with $\mathrm{CPE}$, the importance of preventing CPE transmission has been emphasized $[10,11]$. The Korea Centers for Disease Control and Prevention (KCDC) has been conducting surveillance of $\mathrm{CPE}$ infection/carrier cases. The KCDC expanded the range of surveillance in June 2017, before which sentinel surveillance was performed. However, data on CPE infection in the ROK were limited.

Herein, we aimed to describe the epidemiological, clinical, and microbiological characteristics of CPE BSIs in the ROK. We also aimed to investigate the risk factors for mortality associated with CPE BSIs.

\section{Materials and methods} Study setting and data collection

All CPE isolates obtained from patients at sentinel medical institutions (100 institutions in 2015, and 115 in 2016) were mandatorily reported to the KCDC through the National Surveillance System. An epidemic investigation officer from the KCDC produced epidemic investigation reports on $\mathrm{CPE}$ infection/carrier cases by reviewing patients' medical records including data on clinical features, microbiological data, epidemiological characteristics, and outcomes. We retrospectively reviewed the CPE BSI cases using data collected through the National surveillance system from January 2015 to December 2016, based on the epidemiologic survey performed by the epidemiologist from the KCDC. In particular, we investigated the antibiotic susceptibility of CPE BSI.

\section{Confirmation of CPE}

All sentinel laboratories used automated systems for microorganism identification and in vitro antibiotic susceptibility testing. When sentinel laboratories reported CRE, they also sent samples of specimens containing $\mathrm{CPE}$ to National Institute Health (NIH) in Korea, and the NIH confirmed the presence of CRE. When a sentinel medical institution detected CRE, the isolated specimen was sent to the NIH for confirmatory testing. The NIH performs antibiotic susceptibility tests according to the Clinical and Laboratory Standards Institute guidelines, while resistance is judged based on M100-S27 (2017) [12]. They followed the CDC's "Laboratory Protocol for Detection of Carbapenem-Resistant or Carbapenemase-Producing, Klebsiella spp. and Escherichia coli from Rectal Swabs" [13]. The examination was performed based on the following procedure: all acquired specimens were cultivated in Trypticase soy broth mixed with carbapenem. The cultured fluid was streaked on MacConkey agar and cultivated once more. Then, the isolated colony was determined by phenotype test like mixed hemagglutination test or carbapenem minimum inhibitory concentration (MIC). If carbapenem resistance was confirmed, a carbapenemase gene polymerase chain reaction (PCR) or sequencing was performed to detect the presence of carbapenemase. A modified Hodge test was performed in all the commissioned specimens, and if the result was positive, a genotyping test was performed to confirm the types of carbapenemase.

\section{Definition}

We defined CPE as CRE with any carbapenemase (KPC, NDM, IMP, GES, VIM, or OXA-48), while CRE was defined based on the KCDC guidelines for CPE control, as confirmed by the MIC of each carbapenem: the MIC of doripenem, imipenem, and meropenem was $\geq 4 \mu \mathrm{g} / \mathrm{ml}$, while the MIC of ertapenem was $\geq 2 \mu \mathrm{g} / \mathrm{ml}$ [12].

Hospital-acquired infections were confirmed through the positive blood culture results of patients who were hospitalized for more than $48 \mathrm{~h}$. Community-acquired or healthcare-associated infections were confirmed among patients who had BSI from blood culture samples within $48 \mathrm{~h}$. If they had a history of hospitalization history in the last 90 days or were transferred from another medical institution, the infection was defined as healthcare associated. If the patient developed BSI within $48 \mathrm{~h}$ without history of hospitalization in the last 90 days, community-acquired infection was confirmed.

Empirical therapy was defined as the antimicrobial therapy administered before the bacterial strain was identified and before the susceptibility report was provided by the hospital laboratory [14]. We defined the adequate treatment for $\mathrm{CPE}$ infection if the physician used 
susceptible antibiotics in isolated bacteria in vitro or if the physician chose a combination therapy, at least one drug was active in vitro against the infecting organism [15]. If a clinician used an antibiotic to treat CPE BSI, in which the susceptibility was confirmed by an antibiogram, the antibiotic therapy was judged as being adequate.

Patients who died within 30 days from CPE BSI occurrence were defined as "non-survivors." The clinical data of the patients were collected at the time of CPE BSI detection.

Extended-spectrum beta-lactamase was detected by performing an extended-spectrum beta-lactamase test with disk diffusion or broth microdilution using an automated susceptibility system according to the guidelines of the Clinical and Laboratory Standards Institute [12]. We confirmed ESBLs using automated equipment in each hospital.

\section{Calculation of the proportion of CPE BSI among total CPE isolates}

To calculate the proportion of CPE BSI among CPE isolates, we used the data on total CPE isolates from the Public Health Weekly report, "Status of carbapenemase-producing enterobacteriaceae incidences in Korea, 2015-2016" [6]. This report contains data on the total number of CPE infection/ carrier cases as obtained through national sentinel surveillance, regional occurrences, and microbiological features.

\section{Statistical analysis}

SPSS version 21.0 for Windows (IBM Corporation, Armonk, NY) was used to perform all statistical analyses. Categorical variables were analyzed by a Chi-square test or Fisher's extract test, as appropriate. Continuous variables were analyzed by an independent samples t-test or a Mann-Whitney $U$ test. Logistic regression analysis was performed to evaluate the effect of independent variables on risk. A two-tailed $P$-value of $<0.05$ was considered significant. All variables with $P$ values lower than 0.05 in the univariate analysis were included in the multiple logistic regression models in which we identified the risk factors for mortality due to CPE BSIs.

\section{Results}

\section{Epidemiologic characteristics of CPE BSIs in the ROK}

A total of $2020 \mathrm{CPE}$ isolates were obtained from January 2015 to December 2016. There were 131 cases of CPE BSI (proportion: 6.5\%). Approximately $67 \%$ of the CPE BSI cases were reported in advanced general hospitals. The regional distributions and differences between the microbiological features are described in Table 1. About $48.9 \%$ of the CPE BSI patients were from Seoul; $26.7 \%$ were from Busan, Ulsan, and Gyeongsangnam-do; and $19.8 \%$ were from Incheon and Gyeonggi-do. The proportion of CPE BSI among total CPE isolates were not significantly different between each region and nationwide $(P=0.08$ (Seoul), 0.41 (Busan, Ulsan, and Gyeongsangnam-do), 0.21 (Gyeongangbuk-do and Daegu), 0.63 (Chungcheong-do and Daejeon), 0.21 (Jeolla-do and Gwangju), and 0.64 (Gangwon-do)) except for Incheon and Gyeonggi-do $(P=0.01)$.

Nationwide, the proportion of $K$. pneumoniae BSI was the highest (69\%), followed by that of Enterobacter spp. and $E$. coli infections (10 and $8 \%$, respectively). In the regional analysis, the Enterobacteriaceae types were similar to those observed nationwide. Specifically, Serratia marcescens was prevalent in Busan, Ulsan, and Gyeongsangnam-do (14\%), while Citrobacter spp. was prevalent in Incheon and Gyeonggi-do (8\%). With regard to the types of carbapenemase, KPC was the most commonly observed in the ROK (66\%) followed by NDM (20\%). The patterns of carbapenemase distribution in Seoul were similar to those observed nationwide; however, in Busan, Ulsan, and Gyeongsangnam-do, $\mathrm{KPC}$ was extremely dominant compared with the other types of carbapenemase. In Incheon and Gyeonggi-do, NDM was more dominant than KPC (46 and 42\%, respectively).

Data on infection acquisition are described in Table 2. A total of 111 (85\%) patients had hospital-acquired CPE BSI. About $48(43 \%)$ of the infections occurred during CPE outbreak (data not shown). A total of 15 (12\%) patients had healthcare-associated infections, and 5 (33\%) were transferred from nursing hospitals. About 5 (4\%) patients were suspected of having community-acquired infection. The mode of transmission did not affect mortality $(P=0.08)$.

\section{CPE antibiotic susceptibility}

The antibiotics susceptible to CPE are shown in Table 3 and Additional file 1 Table S1. A total of 99 cases were sensitive to amikacin (75.6\%), 54 were sensitive to tigecycline (41.2\%), and 51 were sensitive to gentamicin (38.9\%). Approximately $47 \%$ of all CPE patients did not have extended-spectrum beta-lactamase (data not shown). Twenty-nine patients from nine medical institutions underwent colistin sensitivity testing, and one patient who was transferred from Dubai, United Arab Emirates, developed colistin resistance.

We demonstrated the antibiotic susceptibility patterns according to the type of Enterobacteriaceae. K. oxytoca was more sensitive to aztreonam, ciprofloxacin/levofloxacin, and cefepime than $K$. pneumoniae $(P<0.001,0.001,<$ 0.001 , and $<0.001$, respectively). S. marcescens was more sensitive to trimethoprim/sulfamethoxazole $(86 \%)$ than other organisms $(P<0.001)$.

When we analyzed the antibiotic susceptibility of $\mathrm{KPC}$-producing CPE or MBL-producing CPE, there was no significant difference in aztreonam susceptibility between KPC and NDM (1.2\% [1/86] in KPC-producing 
Table 1 Epidemic characteristics of carbapenemase-producing Enterobacteriaceae bloodstream infection

\begin{tabular}{|c|c|c|c|c|c|c|c|c|}
\hline & Total & A & B & C & $\mathrm{D}$ & E & $\mathrm{F}$ & G \\
\hline CPE bloodstream infection & 131 & $64(49)$ & $35(27)$ & $26(20)$ & $2(2)$ & $2(2)$ & $1(1)$ & $1(1)$ \\
\hline \multicolumn{9}{|l|}{ Hospital type } \\
\hline General hospital & $43(33)$ & $8(13)$ & $24(69)$ & $8(31)$ & $1(50)$ & $1(50)$ & 0 & $1(100$ \\
\hline Advanced general hospital & $88(67)$ & $56(88)$ & $11(31)$ & $18(69)$ & $1(50)$ & $1(50)$ & $1(100)$ & 0 \\
\hline \multicolumn{9}{|l|}{ Enterobacteriaceae type } \\
\hline Escherichia coli & $10(8)$ & $6(9)$ & $2(6)$ & $1(4)$ & 0 & 0 & $1(100)$ & 0 \\
\hline Klebsiella pneumoniae & $90(69)$ & $42(66)$ & $25(71)$ & $21(81)$ & 0 & $1(50)$ & 0 & $1(100$ \\
\hline Enterobacter species & $13(10)$ & $7(11)$ & $2(6)$ & $2(8)$ & $2(100)$ & 0 & 0 & 0 \\
\hline Klebsiella oxytoca & $7(5)$ & $7(11)$ & 0 & 0 & 0 & 0 & 0 & 0 \\
\hline Citrobacter species & $4(3)$ & $1(2)$ & $1(3)$ & $2(8)$ & 0 & 0 & 0 & 0 \\
\hline Serratia marcescens & $7(5)$ & $1(2)$ & $5(14)$ & 0 & 0 & $1(50)$ & 0 & 0 \\
\hline \multicolumn{9}{|l|}{ Carbapenemase type } \\
\hline OXA-48 & $7(5)$ & $3(5)$ & 0 & $2(8)$ & 0 & 0 & $1(100)$ & $1(100$ \\
\hline NDM & $26(20)$ & $13(20)$ & 1 (3) & $12(46)$ & 0 & 0 & 0 & 0 \\
\hline KPC & $86(66)$ & $40(63)$ & $34(97)$ & $11(42)$ & 0 & $1(50)$ & 0 & 0 \\
\hline VIM & $8(6)$ & $7(11)$ & 0 & 0 & $1(50)$ & 0 & 0 & 0 \\
\hline IMP & $2(2)$ & $1(2)$ & 0 & $1(4)$ & 0 & 0 & 0 & 0 \\
\hline
\end{tabular}

Abbreviations: OXA Oxacillin carbapenemases, NDM New Delhi metallo-beta-lactamase enzyme, KPC Klebsiella pneumoniae Carbapenemase, VIM Verona integronencoded metallo-beta-lactamase, IMP Imipenemase metallo-beta-lactamase, CPE Carbapenemase-producing Enterobacteriaceae Note: Data presented are numbers (\%) of patients, unless otherwise indicated

A: Seoul, B: Busan, Ulsan, and Gyeongsangnam-do, C: Incheon and Gyeonggi-do, D: Gyeongangbuk-do and Daegu, E: Chungcheong-do and Daejeon, F: Jeolla-do and Gwangju, G: Gangwon-do

CPE and 3.8\% [1/26] in NDM-producing CPE, $P=0.41$ ) (Additional file 1 Table S2).

Baseline characteristics of the survivors and non-survivors Table 2 shows the patients' baseline characteristics. The 30-day mortality associated with CPE BSI was $47 \%$. The factors affecting 30-day mortality were presence of lung infection, presence of urinary tract infection, a low Acute Physiology and Chronic Health Evaluation (APACHE) II score, and history of antibiotic therapy for CPE infection $(P=0.02,0.02$, $<0.001$, and 0.03 , respectively).

In the multivariate analysis, the measurement of the APACHE II score at the time of CPE BSI $(P<0.001)$ and provision of adequate antibiotic therapy for $C P E(P=$ 0.01 ) were significantly associated with 30 -day mortality (Table 4).

The application of empirical antibiotics at the occurrence of the first sign of infection did not reduce mortality $(P=0.28)$. However, if physicians applied adequate antibiotics for the treatment of CPE BSI when they recognized that the pathogen had carbapenem resistance, the mortality significantly diminished $(P=0.03)$. The antibiotic regimens used for survivors and non-survivors were not significantly different. Moreover, combination antibiotic therapy did not decrease mortality $(P=0.68)$ (Additional file 1 Table S3).

\section{Discussion}

To our knowledge, this study is the first to demonstrate the epidemiological, microbiological, and clinical features of CPE BSI patients in the ROK. Nationwide, the incidence of $K$. pneumonia with KPC-type carbapenemase was the highest, and most cases originated from hospital-acquired infections. CPE showed sensitivity to amikacin, tigecycline, and gentamicin. In this study, the 30-day mortality was 47\%. A lower APACHE II score at CPE BSI occurrence or the application of adequate antibiotics for CPE was significantly associated with decreased mortality.

CRE is highly prevalent and is considered a public health threat worldwide [16]. According to an annual report of the Korean Antimicrobial Resistance Monitoring System, 2015, $1.7 \%$ of $K$. pneumoniae, $0.3 \%$ of E. coli, and $1.4 \%$ of Enterobacter species are carbapenem resistant, as observed in general hospitals nationwide [17]. Furthermore, the CPE infection incidence rate is increasing every year. According to the data from the KCDC, a total of 16 CPE infection cases were reported in 2011; however, $1455 \mathrm{CPE}$ infection cases were observed in 2016 [6]. Around 40 medical institutions had experienced CPE outbreaks and more than two patients with 
Table 2 Clinical characteristics of carbapenemase-producing Enterobacteriaceae bloodstream infection and antibiotics applied for its treatment

\begin{tabular}{|c|c|c|c|c|}
\hline Characteristics & $\begin{array}{l}\text { Total } \\
(N=131)\end{array}$ & $\begin{array}{l}\text { Survivor } \\
(N=69)\end{array}$ & $\begin{array}{l}\text { Non-survivor } \\
(N=62)\end{array}$ & $P$ \\
\hline Age $\pm S D$ (years) & $59.6 \pm 18.9$ & $57.8 \pm 18.5$ & $61.6 \pm 19.2$ & 0.23 \\
\hline Male & $86(66)$ & $46(67)$ & $40(65)$ & 0.25 \\
\hline Transfer history & $47(36)$ & $24(35)$ & $23(37)$ & 0.93 \\
\hline Acquisition of infection & & & & 0.08 \\
\hline Community & $5(4)$ & $5(7)$ & 0 & 0.06 \\
\hline Healthcare-associated & $15(12)$ & $8(12)$ & $7(11)$ & 0.96 \\
\hline Hospital-acquired & $111(85)$ & $56(81)$ & $55(89)$ & 0.23 \\
\hline \multicolumn{5}{|l|}{ Comorbidity } \\
\hline Total & $112(86)$ & $62(90)$ & $50(81)$ & 0.21 \\
\hline DM & $37(28)$ & $16(23)$ & $21(34)$ & 0.25 \\
\hline Solid organ cancer & $34(26)$ & $18(26)$ & $16(26)$ & 1.00 \\
\hline Hematology cancer & $33(25)$ & $20(29)$ & $13(21)$ & 0.39 \\
\hline ESRD & $21(16)$ & $11(16)$ & $10(16)$ & 1.00 \\
\hline Heart disease & $15(12)$ & $7(10)$ & $8(13)$ & 0.83 \\
\hline Liver disease & $13(9)$ & $7(10)$ & $6(10)$ & 1.00 \\
\hline Stroke & $12(9)$ & $7(10)$ & $5(8)$ & 0.91 \\
\hline Immunosuppressive therapy state & $6(5)$ & $2(3)$ & $4(7)$ & 0.58 \\
\hline COPD & $2(2)$ & 0 & $2(3)$ & 0.43 \\
\hline Charlson comorbidity index & $4.7 \pm 3.0$ & $4.4 \pm 2.8$ & $5.0 \pm 3.3$ & 0.33 \\
\hline \multicolumn{5}{|l|}{ Portal of entry } \\
\hline Pneumonia & $38(29)$ & $14(20)$ & $24(39)$ & 0.02 \\
\hline Primary bacteremia & $21(16)$ & $12(17)$ & $9(15)$ & 0.65 \\
\hline Cholangitis/cholecystitis & $20(15)$ & $11(16)$ & $9(15)$ & 0.82 \\
\hline Skin and soft tissue infection (include post operation site infection) & $14(11)$ & $7(10.1)$ & $7(11.3)$ & 0.83 \\
\hline UTI & $13(10)$ & $11(16)$ & $2(3)$ & 0.02 \\
\hline Unknown & $10(8)$ & $5(7)$ & $5(8)$ & 0.86 \\
\hline Intraabdomen infection & $8(6)$ & $5(7)$ & $3(5)$ & 0.72 \\
\hline catheter-related BSI & $7(5)$ & $4(6)$ & $3(5)$ & 1.00 \\
\hline \multicolumn{5}{|l|}{ Operation } \\
\hline General & $41(31)$ & $17(25)$ & $24(39)$ & 0.12 \\
\hline Minor & $29(22)$ & $13(19)$ & $16(26)$ & 0.45 \\
\hline Simple & $84(64)$ & $41(59)$ & $43(69)$ & 0.32 \\
\hline Tracheostomy & $25(19)$ & $11(16)$ & $14(23)$ & 0.46 \\
\hline Intensive care unit admission & $74(57)$ & $29(42)$ & $45(73)$ & 0.001 \\
\hline Invasive catheter & $124(95)$ & $63(91)$ & $61(98)$ & 0.16 \\
\hline Mechanical ventilation & $60(46)$ & $22(32)$ & $38(61)$ & 0.001 \\
\hline APACHE II score & $17.2 \pm 8.0$ & $12.8 \pm 5.4$ & $22.2 \pm 7.6$ & $<0.001$ \\
\hline \multicolumn{5}{|l|}{ Treatment } \\
\hline Adequate antibiotic therapy & $106(82)$ & $62(90)$ & $46(74)$ & 0.03 \\
\hline Amikacin-based therapy & $33(31)$ & $17(27)$ & $16(38)$ & 0.53 \\
\hline Colistin-based therapy & $58(54)$ & $28(45)$ & $30(65)$ & 0.05 \\
\hline
\end{tabular}

Note: Data presented are numbers (\%) of patients, unless otherwise indicated

Abbreviation: SD Standard deviation, DM Diabetes mellitus, COPD Chronic obstructive pulmonary disease, UTI Urinary tract infection, APACHE Acute

Physiology and Chronic Health Evaluation, CPE Carbapenemase-producing Enterobacteriaceae, TMP/SMX Trimethoprim/sulfamethoxazole 
Table 3 Differences in antibiotic susceptibility by Enterobacteriaceae type

\begin{tabular}{|c|c|c|c|c|c|c|c|c|c|}
\hline \multirow{2}{*}{$\begin{array}{l}\text { Antibiotics } \\
\text { susceptibility }\end{array}$} & \multicolumn{3}{|c|}{ Total $(N=131)$} & \multirow{2}{*}{$\begin{array}{l}\text { Escherichia } \\
\text { coli } \\
(N=10)\end{array}$} & \multirow{2}{*}{$\begin{array}{l}\text { Klebsiella } \\
\text { pneumoniae } \\
(N=90)\end{array}$} & \multirow{2}{*}{$\begin{array}{l}\text { Enterobacter } \\
\text { spp. } \\
(\mathrm{N}=13)\end{array}$} & \multirow{2}{*}{$\begin{array}{l}\text { Klebsiella } \\
\text { oxytoca } \\
(N=7)\end{array}$} & \multirow{2}{*}{$\begin{array}{l}\text { Citrobacter } \\
\text { spp. } \\
(N=4)\end{array}$} & \multirow{2}{*}{$\begin{array}{l}\text { Serratia } \\
\text { marcescen } \\
(N=7)\end{array}$} \\
\hline & Susceptible & Intermediate & Resistant & & & & & & \\
\hline Ampicillin & $1(1)$ & 0 & $130(99)$ & 0 & $1(1)$ & 0 & 0 & 0 & 0 \\
\hline Ampicillin/sulbactam & 0 & 0 & $131(100)$ & 0 & 0 & 0 & 0 & 0 & 0 \\
\hline Amikacin & $99(76)$ & $2(2)$ & $30(23)$ & $9(90)$ & $66(73)$ & $9(69)$ & $6(86)$ & $4(100)$ & $5(71)$ \\
\hline Aztreonam & $6(5)$ & 0 & $125(95)$ & $3(30)$ & 0 & 0 & $3(43)$ & 0 & 0 \\
\hline Ceftazidime & $3(2)$ & 0 & $128(98)$ & 0 & 0 & 0 & $1(14)$ & 0 & $2(29)$ \\
\hline Cefotaxime & $1(1)$ & 0 & $130(99)$ & $1(10)$ & 0 & 0 & 0 & 0 & 0 \\
\hline Ciprofloxacin & $18(14)$ & $3(2)$ & $110(84)$ & $1(10)$ & $7(8)$ & $3(23)$ & $4(57)$ & $2(50)$ & $1(14)$ \\
\hline Cefuroxime & 0 & 0 & $131(100)$ & 0 & 0 & 0 & 0 & 0 & 0 \\
\hline Cefazoline & 0 & 0 & $131(100)$ & 0 & 0 & 0 & 0 & 0 & 0 \\
\hline Cefepime & $8(6)$ & $2(2)$ & $121(92)$ & 0 & $2(2)$ & $2(15)$ & $3(43)$ & 0 & $1(14)$ \\
\hline Cefoxitin & $1(1)$ & 0 & $130(99)$ & 0 & $1(1)$ & 0 & 0 & 0 & 0 \\
\hline Gentamicin & $51(39)$ & $12(9)$ & $68(52)$ & $5(50)$ & $29(32)$ & $5(39)$ & $2(29)$ & $3(75)$ & $7(100)$ \\
\hline Levofloxacin & $12(9)$ & $4(3)$ & $115(88)$ & $1(10)$ & $3(3)$ & $4(31)$ & $3(43)$ & 0 & $1(14)$ \\
\hline Tobramycin & $4(3)$ & $4(3)$ & $123(94)$ & $1(10)$ & $1(1)$ & $2(15)$ & 0 & 0 & 0 \\
\hline Piperacillin/tazobactam & $1(1)$ & $5(4)$ & $125(95)$ & 0 & 0 & 0 & 0 & 0 & $1(14)$ \\
\hline TMP/SMX & $30(23)$ & 0 & $101(77)$ & $2(20)$ & $18(20)$ & $1(8)$ & $2(29)$ & $1(25)$ & $6(86)$ \\
\hline Tigecycline & $54(41)$ & $9(7)$ & $68(52)$ & $7(70)$ & $37(41)$ & $2(15)$ & $4(57)$ & $2(50)$ & $2(29)$ \\
\hline Tetracycline & $12(9)$ & $1(1)$ & $118(90)$ & $1(10)$ & $9(10)$ & $1(8)$ & $1(14)$ & 0 & 0 \\
\hline Ticarcillin/clavulanate & $1(1)$ & $1(1)$ & $129(99)$ & 0 & $1(1)$ & 0 & 0 & 0 & 0 \\
\hline Colistin* & 28/29 (97) & $0 / 29$ & 1/29 (3) & 4/4 (100) & 18/19 (95) & 1/1 (100) & $3 / 3(100)$ & $2 / 2(100)$ & $0 / 0$ \\
\hline
\end{tabular}

Note: Data are numbers (\%) of patients, unless otherwise indicated

Abbreviation: spp. - Species, TMP/SMX - Trimethoprim/sulfamethoxazole

* Only 29 cases were performed colistin sensitivity test

CPE infection/CPE carriers with epidemiological relations were identified.

We found some regional differences in the data on CPE BSI occurrence. This could be attributed to the sentinel medical institutions' characteristics. The number of medical institutions was the greatest in Seoul; moreover, the medical institutions in Seoul were relatively larger than those in the other regions. Therefore, CPE patients were concentrated in Seoul. Some medical institutions experienced large-scale CPE outbreaks in Seoul, Daegu, Busan, and Incheon; these events reflect our total data, including the microbiological features. In particular, the type of CPE, which was popular in most parts of Korea, was a KPC-producing CPE; however, in Incheon, most of the patients had the NDM type. This could be attributed to outbreaks from some medical institutions, and the fact that some CPE types were transmitted between hospitals.

Around 95\% (126/133) of the patients in this study had hospital-acquired or healthcare-associated infections. There have been warnings on CPE outbreaks in medical institutions $[5,18]$. According to the data on the CRE Epicenter of the United States, the rate of hospital-acquired infections was $66 \%$; however, $75 \%$ of the patients who died had hospital-acquired infection
[8]. In this study, $85 \%$ of all CPE BSI patients had hospital-acquired infection; about $43 \%$ of these patients acquired the infection during an outbreak in the hospital. This is why the importance of infection control is emphasized in hospitals.

Understanding the antibiotic susceptibilities of CPE is important for treatment-related decision-making. In the present study, a relatively higher susceptibility with aminoglycosides was noted (amikacin: 76\% and gentamicin: $39 \%$, respectively), which was consistent with those reported in previous studies $[19,20]$. This finding could be attributed to the differences in the characteristics of various carbapenemase types. Similar data were reported by Zubair et al. [21], who analyzed 41 patients in whom KPC-type CPE bacteremia was detected; they found that only $14.6 \%$ of all CPE patients were susceptible to gentamicin. Moreover, NDM types could produce 16S ribosomal RNA methyltransferase, which leads to resistance to all aminoglycosides [22]. In terms of comprehensive treatment options, amikacin combination therapy may be an alternative plan to treat $\mathrm{CPE}$ infection when there is lack of adequate treatment options; however, our data did not reveal the effectiveness of this method in improving survival. More advanced research on the efficacy 
Table 4 Multivariate analysis of the risk factors of mortality due to carbapenemase-producing Enterobacteriaceae bloodstream infection

\begin{tabular}{|c|c|c|c|c|}
\hline \multirow[t]{2}{*}{ Characteristics } & \multicolumn{2}{|c|}{ Univariate analysis } & \multicolumn{2}{|c|}{ Multivariate analysis } \\
\hline & $\begin{array}{l}\text { Unadjusted } \\
\text { OR (95\% Cl) }\end{array}$ & P & $\begin{array}{l}\text { Adjusted } \\
\text { OR (95\% CI) }\end{array}$ & P \\
\hline Age & $1.01(0.99-1.03)$ & 0.23 & - & - \\
\hline Pneumonia & $2.48(1.14-5.40)$ & 0.02 & - & - \\
\hline UTI & $0.18(0.03-0.83)$ & 0.02 & - & - \\
\hline Community-acquired infection & $0.93(0.87-0.99)$ & 0.06 & - & - \\
\hline ICU admission & $3.65(1.75-7.61)$ & 0.001 & - & - \\
\hline General operation & $1.93(0.91-4.09)$ & 0.08 & - & - \\
\hline Mechanical ventilation & $3.38(1.65-6.95)$ & 0.001 & - & - \\
\hline APACHE II score & $1.25(1.16-1.35)$ & $<0.001$ & $1.23(1.13-1.34)$ & $<0.001$ \\
\hline Antibiotics therapy for CPE infection & $0.27(0.09-0.75)$ & 0.03 & $0.12(0.03-0.57)$ & 0.007 \\
\hline Colistin based therapy & $1.29(0.65-2.58)$ & 0.05 & - & - \\
\hline Combination therapy & $0.54(0.24-1.25)$ & 0.15 & - & - \\
\hline
\end{tabular}

Abbreviation: OR Odd ratio, ICU Intensive Care Unit, UTI Urinary tract infection, APACHE Acute Physiology and Chronic Health Evaluation, CPE Carbapenemaseproducing Enterobacteriaceae, No. Number, Cl Confidential index

of amikacin combination treatment for CPE BSI should be performed.

Previous studies described the risk factors for mortality due to $\mathrm{CPE}$ infection to predict treatment outcomes. Mario et al. stated that the occurrence of septic shock, inadequate initial antimicrobial treatment, and a high APACHE II score increased mortality in patients with CPE BSI, but the application of combination therapy for CPE infection could decrease mortality [23]. Our data also suggested that patients' underlying conditions including their APACHE II scores could be associated with mortality; however, the difference in mortality between different antibiotic regimens was small. This could be because patients with higher disease severity may have received combination therapy, compared with patients with mild-to-moderate disease. Most monotherapy regimens include colistin, which many CPE patients are susceptible; this could affect the survival of CPE BSI patients.

The strength of our study is that it is the first and largest research in the ROK to obtain data regarding the epidemiology, microbiology, and clinical features, including antibiotic susceptibility, of CPE bacteremia cases. However, our study has some limitations. First, as we used the data from epidemic investigation reports, only limited clinical data were obtained, such as duration of antibiotic therapy or data on why clinicians chose a particular antibiotic. Second, our data were obtained from sentinel surveillance. We only included those hospitals capable of monitoring and managing infectious diseases. Therefore, our findings cannot be generalized to smaller-sized hospital or clinics. Third, although most CPE BSI infections were hospital-acquired or healthcare-associated infections $(96 \%, 126 / 131)$, we could not determine the carriage rate of CPE in patients with
CPE BSI upon admission. In a previous study conducted between May 2016 and February 2017, in similar periods with that of our study in ROK, the CRE carriage rate of patients who transferred from long-term care facilities was 1.4\% (4/282) without CPE colonizer [24]. Moreover, about one-third (37\%) of the total CPE BSI cases occurred in the outbreak setting (data not shown). This finding suggests that most patients with CPE BSI currently have low CPE carriage rate. However, due to the rapidly changing $\mathrm{CPE}$ epidemiology in ROK, it is important to apply efficient CPE screening strategy in inpatients according to each institution's epidemiology [25]. Fourth, carbapenem-sensitive or non-susceptible CPE may have been missed since the focus is on testing for presence of carbapenemase genes on carbapenem-resistant isolates only. This may underestimate the burden of CPE BSI.

\section{Conclusions}

We found that the proportion of CPE BSI per total CPE isolates was high, nationwide, and most of these infections were transmitted in hospitals. The establishment of appropriate infection control strategies for medical institutions is important. Moreover, the application of adequate antibiotics including amikacin, gentamicin, or tigecycline could decrease the mortality associated with CPE BSI. More advanced research should be conducted in this regard.

\section{Additional file}

Additional file 1: Table S1. Antibiotics susceptibility test depending on carbapenemase types. Table S2. Comparison of antibiotics susceptibility test between KPC and NDM. Table S3. Antibiotics regimen for treating carbapenemase-producing Enterobacteriaceae bloodstream infection. (DOCX $30 \mathrm{~kb}$ ) 


\section{Abbreviations}

BSI: Bloodstream infection; CPE: Carbapenemase-producing Enterobacteriaceae; KCDC: Korea Centers for Disease Control and Prevention; MIC: Minimum inhibitory concentration; NIH: National Institute Health; ROK: Republic of Korea

\section{Acknowledgements}

We thank SJ Lee and HK Kim for having received epidemiologic data of CPE $\mathrm{BSI}$ cases. We also thank each of the infection control teams in the medical institutions that were included in the sentinel surveillance that provided the clinical data of cases.

\section{Disclaimers}

The opinions expressed by the authors contributing to this journal do not necessarily reflect the opinions of the Centers for Disease Control and Prevention or the institutions with which the authors are affiliated.

\section{Funding}

This research was supported by the Soonchunhyang University Research Fund.

\section{Availability of data and materials}

Data sharing is not applicable to this article as no datasets were generated or analyzed in the current study.

\section{Authors' contributions}

PJW and PSY designed the study. PJW and LH collected the data. PJW, PSY, and KTH analyzed and interpreted the data. PJW, LH, and PSY wrote the manuscript. All authors read and approved the final manuscript.

\section{Authors' information}

JW Park is a medical doctor at Asan Medical Center, Department of Infectious Disease, and worked with epidemic investigation officer in KCDC. $\mathrm{LH}$ is medical doctor majored in preventive medicine and the government officer in division of Healthcare Associated Infection Control, KCDC.

\section{Ethics approval and consent to participate}

The study protocol was approved by the institutional review board of the KCDC (2018-04-01-PE-A), and the requirement for written informed consent from patients was waived.

\section{Consent for publication}

Not applicable.

\section{Competing interests}

I declare that I have no significant competing financial, professional, or personal interests that might have influenced the performance or presentation of the work described in this manuscript.

\section{Publisher's Note}

Springer Nature remains neutral with regard to jurisdictional claims in published maps and institutional affiliations.

\footnotetext{
Author details

${ }^{1}$ Department of Infectious Disease, Asan Medical Center, University of Ulsan College of Medicine, 88, Olympic-ro 43-gil, Songpa-gu, Seoul 05505, Republic of Korea. ${ }^{2}$ Department of Healthcare Associated Infection Control, Korea Centers for Disease Control and Prevention, 187, Osongsaengmyeong 2-ro, Osong-eup, Heungdeok-gu, Cheongju-si, Chungcheongbuk-do 28159, Republic of Korea. ${ }^{3}$ Division of Infectious Diseases, Department of Internal Medicine, Soonchunhyang University Seoul Hospital, Soonchunhyang University College of Medicine, 59, Daesagwan-ro, Yongsan-gu, Seoul 04401, Republic of Korea.
}

Received: 5 September 2018 Accepted: 13 February 2019 Published online: 05 March 2019

\section{References}

1. Trubiano JA, Padiglione AA. Nosocomial infections in the intensive care unit. Anaesth Intensive Care Med. 2015;16:598-602.
2. Mitharwal SM, Yaddanapudi S, Bhardwaj N, Gautam V, Biswal M, Yaddanapudi L. Intensive care unit-acquired infections in a tertiary care hospital: an epidemiologic survey and influence on patient outcomes. Am J Infect Control. 2016;44:e113-7.

3. Sligl WI, Dragan T, Smith SW. Nosocomial gram-negative bacteremia in intensive care: epidemiology, antimicrobial susceptibilities, and outcomes. Int Journal Infect Dis. 2015;37:129-34.

4. Zilberberg MD, Shorr AF, Micek ST, Vazquez-Guillamet C, Kollef MH. Multi-drug resistance, inappropriate initial antibiotic therapy and mortality in gram-negative severe sepsis and septic shock: a retrospective cohort study. Crit Care. 2014;18:596.

5. Hong SK, Yong D, Kim K, Hong SS, Hong SG, Khosbayar T, et al. First outbreak of KPC-2-producing Klebsiella pneumoniae sequence type 258 in a hospital in South Korea. J Clin Microbiol. 2013;51:3877-9.

6. Park JW LE, Lee SJ, Lee H. Status of carbapenemase-producing Enterobacteriaceae incidences in Korea, 2015-2016. Public Health Weekly Report, KCDC 2017:10:1243-1247.

7. Giske CG, Monnet DL, Cars O, Carmeli Y. Clinical and economic impact of common multidrug-resistant gram-negative bacilli. Antimicrob Agents Chemother. 2008;52:813-21.

8. Satlin MJ, Chen L, Patel G, Gomez-Simmonds A, Weston G, Kim AC, et al. Multicenter clinical and molecular epidemiological analysis of bacteremia due to carbapenem-resistant Enterobacteriaceae (CRE) in the CRE epicenter of the United States. Antimicrob Agents Chemother. 2017;61.

9. Tamma PD, Goodman KE, Harris AD, Tekle T, Roberts A, Taiwo A, et al. Comparing the outcomes of patients with carbapenemase-producing and non-carbapenemase-producing carbapenem-resistant Enterobacteriaceae bacteremia. Clin Infect Dis. 2017;64:257-64.

10. Gupta N, Limbago BM, Patel JB, Kallen AJ. Carbapenem-resistant Enterobacteriaceae: epidemiology and prevention. Clin Infect Dis. 2011; 53:60-7.

11. Schwaber MJ, Carmeli Y. Carbapenem-resistant Enterobacteriaceae: a potential threat. JAMA. 2008:300:2911-3.

12. Patel JB. Performance standards for antimicrobial susceptibility testing: clinical and laboratory standards institute; 2017.

13. Laboratory protocol for detection of carbapenem-resistant or carbapenemase-producing, Klebsiella spp. and E. coli from rectal swabs. CDC, Atlanta, GA. 2009.

14. Villegas MV, Pallares CJ, Escandon-Vargas K, Hernandez-Gomez C, Correa A, Alvarez C, et al. Characterization and clinical impact of bloodstream infection caused by carbapenemase-producing Enterobacteriaceae in seven Latin American countries. PLoS One. 2016;11:e0154092.

15. Tzouvelekis LS, Markogiannakis A, Piperaki E, Souli M, Daikos GL. Treating infections caused by carbapenemase-producing Enterobacteriaceae. Clin Microbiol Infect. 2014;20:862-72.

16. Nordmann P, Cuzon G, Naas T. The real threat of Klebsiella pneumoniae carbapenemase-producing bacteria. Lancet Infect Dis. 2009;9:228-36.

17. National Institute of Health K. Korean antimicrobial resistance monitoring system; 2015.

18. Samra Z, Ofir O, Lishtzinsky Y, Madar-Shapiro L, Bishara J. Outbreak of carbapenem-resistant Klebsiella pneumoniae-producing KPC-3 in a tertiary medical Centre in Israel. Int J Antimicrob Agents. 2007;30:525-9.

19. Guh AY, Bulens SN, Mu Y, Jacob JT, Reno J, Scott J, et al. Epidemiology of carbapenem-resistant Enterobacteriaceae in 7 US communities, 20122013. JAMA. 2015;314:1479-87.

20. Findlay J, Hopkins KL, Alvarez-Buylla A, Meunier D, Mustafa N, Hill R, et al. Characterization of carbapenemase-producing Enterobacteriaceae in the west midlands region of England: 2007-14. J Antimicrob Chemother. 2017;72:1054-62.

21. Qureshi ZA, Paterson DL, Potoski BA, Kilayko MC, Sandovsky G, Sordillo

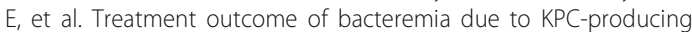
Klebsiella pneumoniae: superiority of combination antimicrobial regimens. Antimicrob Agents Chemother. 2012;56:2108-13.

22. Doi Y, Paterson DL. Carbapenemase-producing Enterobacteriaceae. Seminars in respiratory and critical care medicine. NIH Public Access. 2015;2015:74.

23. Tumbarello M, Viale P, Viscoli C, Trecarichi EM, Tumietto F, Marchese A, et al. Predictors of mortality in bloodstream infections caused by Klebsiella pneumoniae carbapenemase-producing K. pneumoniae: importance of combination therapy. Clin Infect Dis. 2012;55:943-50. 
24. Hwang JH, Park JS, Lee E, et al. Active surveillance for carbapenem-resistant Enterobacteriaceae, vancomycin-resistant Enterococci and toxigenic

Clostridium difficile among patients transferred from long-term care facilities in Korea. J Hosp Infect. 2018;99:487-91.

25. Otler JA, Dyakova E, Bisnauthsing KN, et al. Universal hospital admission screening for carbapenemase-producing organisms in a low prevalence setting. J Antimicrob Chemother. 2016;71:3556-61.

Ready to submit your research? Choose BMC and benefit from:

- fast, convenient online submission

- thorough peer review by experienced researchers in your field

- rapid publication on acceptance

- support for research data, including large and complex data types

- gold Open Access which fosters wider collaboration and increased citations

- maximum visibility for your research: over $100 \mathrm{M}$ website views per year

At BMC, research is always in progress.

Learn more biomedcentral.com/submissions 